\title{
Effect of Coronal Mass Ejection Interactions on the SOHO/CELIAS/MTOF Measurements
}

\author{
X. Wang ${ }^{1}$, P. Wurz ${ }^{1}$, P. Bochsler ${ }^{1}$ \\ F. Ipavich ${ }^{2}$, J. Paquette ${ }^{2}$ and R.F. Wimmer-Schweingruber ${ }^{3}$ \\ ${ }^{1}$ Physics Institute, University of Bern, 3012 Bern, Switzerland \\ email: xuyu.wang@soho.unibe.ch \\ ${ }^{2}$ University of Maryland, College Park, MD20742, USA \\ email: ipavich@umtof.umd.edu \\ ${ }^{3}$ Extraterrestrische Physik, University of Kiel, 24098 Kiel, Germany \\ email: wimmer@physik.uni-kiel.de
}

\begin{abstract}
By using the plasma composition data from SOHO/CELIAS/MTOF, charge states data from ACE/SWICS, combining with the remote sensing observations from SOHO/LASCO white-light image and WIND/WAVES radio emission, we describe a coronal mass ejection (CME) observed on 2001 October 19 16:50 UT to show how the effect of CME interaction appears in the in situ measurements. A new narrow shock is formed while the rear CME passing through the core region of the preceding one, which moves faster than the surrounding part and has a new type II radio burst associated with it. Because of its distinguished elemental abundance and unusual low charge states, we connect a density hump observed by MTOF/PM with the preceding CME core. By comparing the relative abundances of minor ions in shock compressive region, ICME region and CME core region with respect to that in upstream slow solar wind, we indicate mass-per-charge dependence of minor thermal ions may be an important imprint of the characteristic velocity of distant acceleration region.
\end{abstract}

Keywords. shock waves, Sun: abundances, coronal mass ejections (CMEs) solar wind, radio radiation

\section{Introduction}

Our current knowledge of CME comes from two spatial domains: the near-Sun (up to $32 \mathrm{Rs}$ ) region remote-sensed by coronagraphs and the geospace and beyond where in situ observations are made by spacecraft. Comparing observations from these two domains helps us understand the propagation and evolution of CME through the interplanetary (IP) medium. The CME interaction has important implications for the space weather prediction, for instance, some of the false alarms or complex ejecta with unusual composition could be accounted for by CME interactions (Gopalswamy et al., 2001). The question then arises as to how the plasma composition reflects the CME interaction in the in situ measurements. In other words, how does the characteristic velocity of acceleration region show up in the in situ measurements? How to fit the distant observations comparing with the in situ measurements? What can we learn from distant observations of shocks? Can the correlation study of the compressive region with upstream of shock in the in situ measurements be a tracer of the strength of CME interaction?

The purpose of this work is to extend our understanding of shock formation and propagation in the solar wind to the outer coronal region by separating the accelerating effects of IP shock transport and the amplified waves near the Sun on thermal ions. 
Compared with energetic particles, thermal ions have advantages to address the injection efficiency of distant shock because their dispersion and transport process are not strong to wash out the initial injection signature. For thermal ions, the shock acceleration efficiency of the observed particles and resonant wave intensities both peak near the time of shock. Acceleration remains strong as shock propagates in IP medium. Therefore, thermal ions take both the information of the distant amplified waves and the IP transport. In order to separate them, we make two assumptions: first, the primary IP transport is a cascading process; second, the cascading process leads to similar features of $\mathrm{m} / \mathrm{q}$ dependence to downstream particles. The distinguished features relying on the plasma conditions would reflect the injection conditions of shocks, since Mach number and plasma beta are the most important factors to determine the shock injection efficiency. They strongly reply on the local plasma conditions rather than the solar wind speed in the region before the Alfvén point where most CME interactions occur. Therefore, $\mathrm{m} / \mathrm{q}$ dependence of thermal ions must take some imprints of CME interaction. In the CME interaction, the rear $\mathrm{CME}$ has to pass through the core, cavity and frontal of the preceding CME. We address the effects of CME interaction in these inhomogeneous regions by comparing the $\mathrm{m} / \mathrm{q}$ dependence of their correlation to the upstream solar wind.

We analyze a CME event observed on 2001 October 19 16:50 UT by using the observations of SOHO/CELIAS/MTOF plasma composition, ACE/SWICS charge state, $\mathrm{SOHO} / \mathrm{LASCO}$ white image, and WIND/WAVES radio emission. First, we report on a new type II spectral feature for CME interaction, then provide the evidences to connect a density hump observed by MTOF/PM with the preceding CME core and show how the effect of CME-interaction appears in the $\mathrm{m} / \mathrm{q}$ dependence of the correlation of the shock compressive region and CME core region with upstream solar wind, and finally discuss the implications of varied shock strength on thermal ions.

\section{Data and Analysis}

The general population of CMEs observed by SOHO/LASCO is listed in the on-line catalog (http://cdaw.gsfc.nasa.gov). (For the LASCO instrument, see the review paper of Brueckner et al., 1995). The halo CME onset at 2001 October 19 16:50 UT interacts with a narrow slow CME onset about 7 hours earlier with a speed $466 \mathrm{~km} / \mathrm{s}$, central positionangle $241^{\circ}$, width $37^{\circ}$, accelerating rate $8.4 \mathrm{~km} / \mathrm{s}^{2}$. The interaction region is located in the field view of LASCO/C3 coronagraph. The image reveals that both CMEs originate from the west front side of solar disk, the preceding one is on the south (S30), the rear one is on the north (N40). So the interaction we observed in the coronagraph images should not be a superposition in the sky plane. The shock front of the rear CME shows different behavior when it passes through the core of the preceding CME at around 18:18 UT. It moves faster than the surrounding part (see the right image of figure 1). At nearly the same time, WIND/WAVES RAD1 and RAD2 radio receivers detected a sudden enhancement of type II emission at the frequency starting at $1200 \mathrm{kHz}$ and drifting to $250 \mathrm{kHz}$ (For the WAVES experiment, see the paper of Bougeret et al., 1995). The left image of figure 1 shows the dynamic spectrum of the event from 16:30 UT to 21:20 UT. No other feature in the LASCO field of view can be identified as an alternative source of this radio enhancement. Therefore we connect these two phenomena to each other. A possible explanation of this type II burst is: when a fast mode shock front is refracted into a low Alfvén velocity region and the shock strength built up above a certain critical value because of a wave focusing effect. This leads to a concentration of energy favorable to the acceleration of particles in the shock front, resulting in a type II event. 

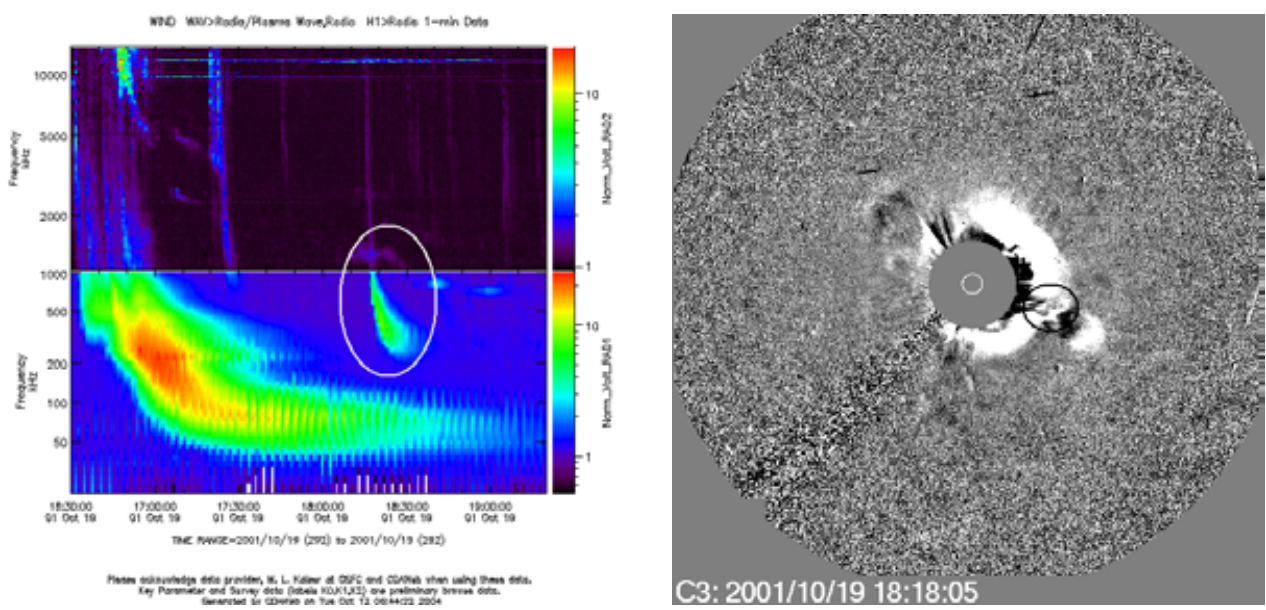

Figure 1. The WIND/WAVES dynamic spectrum on October 19, 2001 from 16:30 UT to 21:20 UT and SOHO/LASCO/C3 image on 2001/10/19 18:18:05 UT when the rear CME passing through the core of the preceding one. The circles indicate the corresponding of a type II emission to the CME interaction.

The MTOF sensor of CELIAS on board SOHO is an isochronous time-of-flight mass spectrometer with a resolution $\mathrm{M} / \Delta \mathrm{M}$ better than 100 and a temporal resolution of 5 min. It detects ions at solar wind bulk velocities of $300-1000 \mathrm{~km} / \mathrm{s}$, corresponding to the energy of about $0.3-3 \mathrm{keV} / \mathrm{amu}$. A sub-sensor of MTOF, Proton Monitor, is used to select appropriate solar wind time intervals for study (Ipavich et al., 1998). A detail description of the CELIAS instrument is given by Hovestadt et al. (1995). Another input parameter for determining the MTOF sensor response is the charge-state distribution. The charge-state distribution measured by ACE/SWICS instrument for O, Mg, Si, Fe are used to interpolate the charge state distribution of other elements. (Wurz, 1999; Wurz et al., 2000; Mazzotta et al., 1998).

We identified the ICME and its corresponding CME by referring to the paper of Cane and Richardson (2003). Figure 2 (left) shows the solar wind plasma parameters and mean charge states (O, Mg, Si, Fe) measured by CELIAS/MTOF/PM and ACE/SWICS, respectively. The investigated time periods are indicated in the top four panels. The CME core period with unusual low charge states corresponds to a density hump detected by $\mathrm{MTOF} / \mathrm{PM}$ with a little time shift attributed to the different locations of the SOHO and ACE spacecraft. Figure 2 (right) shows the double ratio of the abundance in the shock compressive region, ICME region, and CME core region to the referenced upstream abundance of an element $\mathrm{X}$ relative to oxygen $\left([X / O] /[X / O]_{\text {ref }}\right)$ as a function of the mass per charge. Except the element $\mathrm{Si}$ (we omit this point because there is only one measured value in that core region without statistical error), the compressive region and CME core region correlated with the upstream solar wind with positive $\mathrm{m} / \mathrm{q}$ dependence, and no positive $\mathrm{m} / \mathrm{q}$ dependence in the ICME region.

\section{Discussion}

When the Mach number of the upstream flow is increased, the magnetic profile of the shock would change: a magnetic foot develops upstream of the main shock ramp and an overshoot appears at the top of the ramp. The changes in magnetic profile are closely associated with the reflected ions at the shock front, which amplify the magnitude of the waves near the shock and provide a necessary additional dissipation to the particles. 

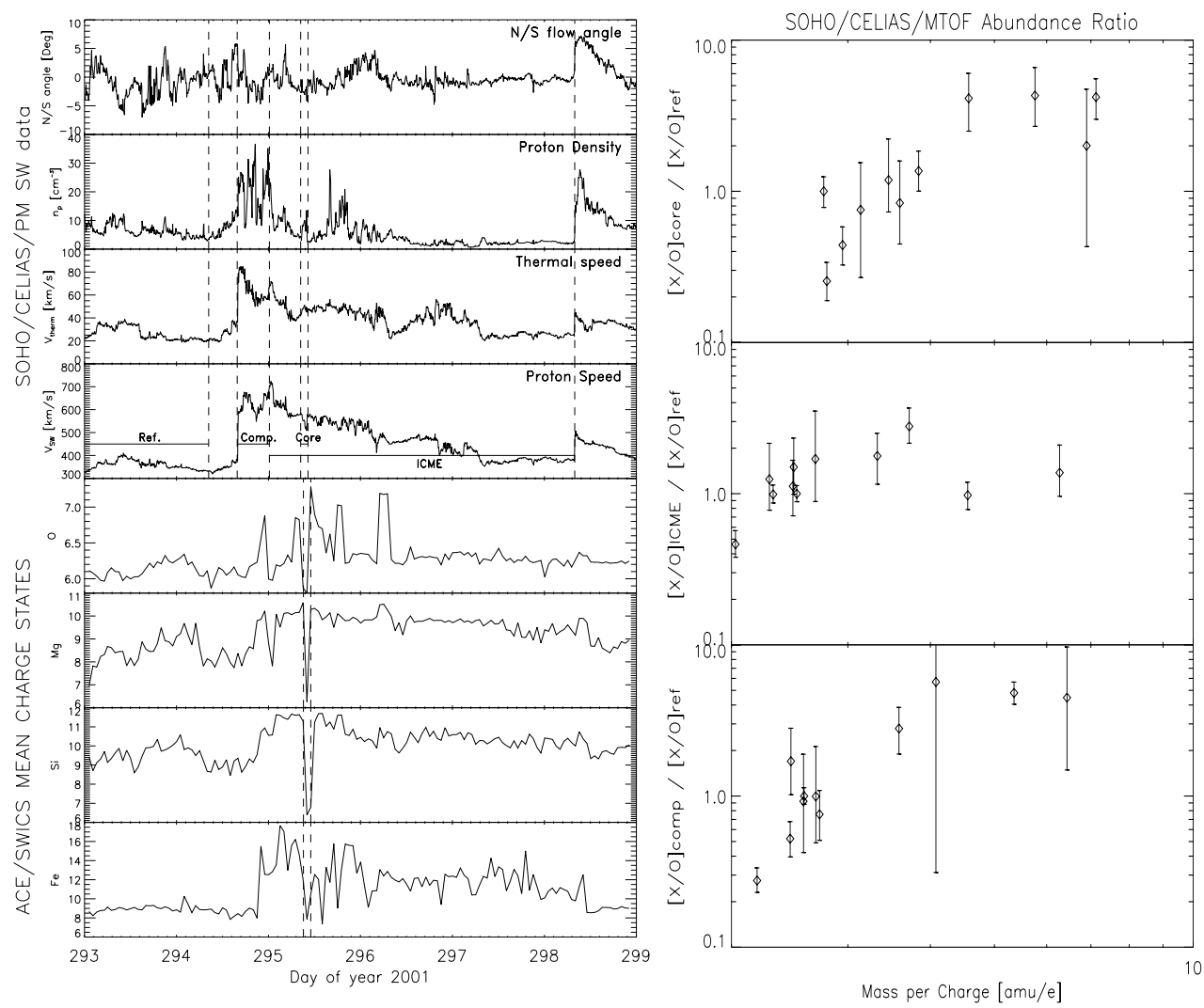

Figure 2. Left: The solar wind plasma parameters and mean charge states $(\mathrm{O}, \mathrm{Mg}, \mathrm{Si}, \mathrm{Fe})$ measured by CELIAS/MTOF/PM and ACE/SWICS, respectively. Right: The double ratio of the abundance for the time periods of shock compressive, ICME, and CME core to the referenced upstream abundance of an element X relative to oxygen $\left([X / O] /[X / O]_{r e f}\right)$ as a function of the mass per charge. The error bars include statistical and instrumental errors.

Therefore, the strengthened shock would lower the threshold of the injection velocity and feed more energy to low-rigidity particles with an enhanced the acceleration efficiency. The distant amplified MHD waves can't be remotely sensed. They leave some observable imprints on the solar energetic particles (SEPs) in several ways, such as limit intensity of SEPs (Reames and Ng, 1998), time variations of SEP elemental abundances (Tylka, Reames and NG, 1999). To the thermal ions, turbulence effect would show up when the energy is below the threshold energy of shock acceleration by the positive $\mathrm{m} / \mathrm{q}$ dependence of elemental abundances in correlation with the upstream solar wind. As we see in the event of Oct. 19, 2001, positive m/q dependences are shown in the shock compressive region and $\mathrm{CME}$ core region due to the $\mathrm{CME}$ interaction. We are looking at this point by checking more CME interaction events with different initial speed, interaction width, etc.

Once the energy of particles reach the threshold energy of shock acceleration, turbulence effect would be covered by the shock acceleration. In an ideal situation, we would expect relatively flat $\mathrm{m} / \mathrm{q}$ dependence. So far the origin and the injection of the seed particles of IP shocks is not well resolved. There is considerable evidence from heliospheric shock observations and plasma simulations that shocks can directly accelerate thermal particles (Forman and Webb, 1985; Gosling, 1993), although Klecker et al. (1981) and 
Tan et al. (1989) interpreted their results in terms of shock acceleration of ions left over from previous energetic particle events. Seeking the energy channel with flat m/q dependence would be significant to address the problem of the threshold energy of shock acceleration.

\section{Acknowledgements}

The CME catalog is generated and maintained by NASA and The Catholic University of America in cooperation with the Naval Research Laboratory. SOHO is a project of international cooperation between ESA and NASA. The dynamic spectrum is provided by M. L. Kalear at GSFC and CDAWeb. This work is supported by the Swiss National Science Foundation.

\section{References}

Mazzotta, P., Mazzitelli, G., Colafrancesco, S., \& Vittorio, N. 1998, Astron. Astrophys. Suppl. Ser. 133,403

Bougeret, J. L., Kaiser, M. L., Kellogg, P. J., Manning, R., Goetz, K., Monson, S. J., Monge, N., Friel, L., Meetre, C. A., Perche, C., Sitruk, L., \& Hoang S. 1995, Space Sci. Rev. 71, 231

Brueckner, G. E., Howard, R. A., Koomen, M. J., Korendyke, C. M., Michels, D. J., Moses, J. D., Socker, D. G., Dere, K. P., Lamy, P. L., Llebaria, A., Bout, M. V., Schwenn, R., Simnett, G. M., Bedford, D. K., \& Eyles, C. J. 1995, Sol. Phys. 162, 357

Forman, M. A. \& Webb, G. M. 1985, in Collisionless Shocks in the Heliosphere: Reviews of current Research, ed. B. T. Tsurutani and R. G. Stone 91

Gopalswamy, N., Yashiro, S., Kaiser, M. L., Howard, R. A., \& Bougeret, J. L. 2001, Astrophys. J. 548, L91

Gosling, J. T. 1993, J. Geophys. Res. 98, 18937

Hovestadt, D., Hilchenbach, M., Bürgi, A., Klecker, B., Laeverenz, P., Scholer, M., Grünwaldt, H., Axford, W. I., Livi, S., Marsch, E., Wilken, B., Winterhoff, H. P., Ipavich, F. M., Bedini, P., Coplan, M. A., Galvin, A. B., Gloeckler, G., Bochsler, P., Balsiger, H., Fischer, J., Geiss, J., Kallenbach, R., Wurz, P., Reiche, K.-U., Gliem, F., Judge, D. J., Ogawa, H. S., Hsieh, K. C., Moebius, E., Lee, M. A., Managadze, G. G., Verigin, M. I., \& Neugebauer, M. 1995, Sol. Phys. 162, 441

Ipavich, F. M., Galvin, A. B., Lasley, S. E., Paquette, J. A., Hefti, S., Reiche, K.-U., Coplan, M. A., Gloeckler, G., Bochsler, P., Hovestadt, D., Grünwaldt, H., Hilchenbach, M., Gliem, F., Axford, W. I., Balsiger, H., Bürgi, A., Geiss, J., Hsieh, K. C., Kallenbach, R., Klecker, B., Lee, M. A., Mangadze, G. G., Marsch, E., Möbius, E., Neugebauer, M., Scholer, M., Verigin, M. I., Wilken, B., \& Wurz P. 1993, J. Geophys. Res. 103, 17205

Klecker, B., Scholer, M., Hovestadt, D., Gloeckler, G., \& Ipavich, F. M. 1981, Astrophys. J. 251, 393

Reames, D. V. \& Ng, C. K. 1998, Astrophys. J. 504, 1002

Tan, L. C., Mason, G. M. Klecker, B., \& Hovestadt, D. 1989, Astrophys. J. 345, 572

Tylka, A. J., Reames, D. V., \& Ng, C. K. 1999, Geophys. Res. Lett. 26, 2141

Wurz, P. 1999, Habilitation thesis, University of Bern, Bern, Switzerland

Wurz, P., Wimmer-Schweingruber, R. F., Bochsler, P., Galvin, A. B., Paquette, J. A., Ipavich, F. M., \& Gloeckler, G. 2001, AIP on Solar and Galactic Composition CP-598, 145 